\title{
Correction to: Low-dose radiation from A- bombs elongated lifespan and reduced cancer mortality relative to un-irradiated individuals
}

Shizuyo Sutou

\section{Correction: Genes Environ. \\ https://doi.org/10.1186/s41021-018-0114-3}

In the original publication of this article [1], the author pointed out the reference list mismatched citations in the main text. All citations and references have been updated.

In Table 1, the data is in incorrect volume. The correct table is below.

The publisher apologizes to the readers and author for the inconvenience.

The original publication has been corrected.

Received: 19 March 2019 Accepted: 20 March 2019

Published online: 19 April 2019

\section{Reference}

1. Sutou S. Low-dose radiation from A-bombs elongated lifespan and reduced cancer mortality relative to un-irradiated individuals. Genes Environ. 2018;40: 26. https://doi.org/10.1186/s41021-018-0114-3

2. Vital Statistics Japan Ministry of Health, Labour, and Welfare (cancer_ mortality(1958- 016).xls in https://ganjoho.jp/reg_stat/statistics/dl/index.html. Accessed 1 Oct 2018

3. Preston DL, Ron E, Tokuoka S, Funamoto S, Nishi N, Soda M, et al. Solid cancer incidence in atomic bomb survivors: 1958-1998. Radiat Res. 2007;168: $1-64$.

4. Ozasa K, Shimizu Y, Suyama A, Kasagi F, Soda M, Grant EJ, et al. Studies of the mortality of atomic bomb survivors, report 14, 1950-2003: an overview of cancer and non cancer diseases. Radiat Res. 2012;177:229-43.

5. Grant EJ, Brenner A, Sugiyama H, Sakata R, Sadakane A, Utada M, et al. Solid Cancer incidence among the life span study of atomic bomb survivors: 1958-2009. Radiat Res. 2017;187:513-37. 
Table 1 Comparison of solid cancer mortality in the lifespan study of A-bomb survivors with Japanese cancer mortality. Japanese average cancer deaths were calculated by dividing cancer deaths by total deaths each year during 1958-2009 [2]. Averages corresponding to survey periods were determined

\begin{tabular}{|c|c|c|c|c|c|}
\hline Reporters & Year & Survey period & No. hibakusha or [NIC $\left.{ }^{\mathrm{a}}\right]$ & No. cancer deaths (\%) & \% Japanese average cancer deaths \\
\hline \multirow[t]{2}{*}{ Preston et al. [3] } & 2007 & 1958-1998 & 105,427 & $17,448(16.6)$ & $21.4(1958-1998)$ \\
\hline & & & {$[25,427]$} & {$[3,994(15.7)]$} & \\
\hline \multirow[t]{2}{*}{ Ozasa et al. [4] } & 2012 & 1958-200 & 86,611 & $10,929(12.6)$ & $22.3(1958-2003)$ \\
\hline & & & {$[26,529]$} & {$\left[N A^{b}\right]$} & \\
\hline \multirow[t]{2}{*}{ Grant et al. [5] } & 2017 & 1958-2009 & 80,205 & $17,316(21.5)$ & 23.3 (1958-2009) \\
\hline & & & {$[25,239]$} & {$[5,222(20.6)]$} & \\
\hline
\end{tabular}

${ }^{a}$ not in the city; ${ }^{b}$ not available 